Original Article

\title{
Urinary tract infections by multidrug resistant Acinetobacter species-A Retrospective Analysis
}

\author{
Prashant Mule', Niranjan Patil', Seema Gaikwad ${ }^{3}$ \\ ${ }^{1,2,3}$ Consultant Microbiologist, Dept of Microbiology and Molecular Microbiology, Metropolis Healthcare Ltd, M umbai, \\ India- 400070.
}

Corresponding Author : Prashant Mule, M icrobiologist, Room No 29, G B Pant Hospital, Atlanta Point, Port Blair, Dist. - South Andaman, Andaman and Nicobar Islands, India- 744 104, M obile : 9180874 66641, E-mail : prashant.mule88@ gmail.com

$\begin{array}{ll}\text { Received } & : 08.01 .2018 \\ \text { Review Completed } & : 13.04 .2018 \\ \text { Accepted } & : 10.06 .2018\end{array}$

Keywords: Nosocomial infections, Acinetobacter baumannii complex, MALDI-TOF MS, Urinary

\begin{tabular}{|c|}
\hline Access this article online \\
\hline Quick Response Code \\
\hline \\
\end{tabular}
catheterization.

\begin{abstract}
Background : Acinetobacter species have emerged as important cause of nosocomial infections like pneumonia and urinary tract infections. Acinetobacter species are known to be highly resistant to commonly used antimicrobial agents. SinceAcinetobacter species are ubiquitous and have tremendous colonizing capacity, it is difficult to explain the role of Acinetobacter acquisition in the ICU and medical wards. The objective of this study was to determine the frequency of urinary tract infection (UTI) caused by different Acinetobacter species in hospitalized patients and in the community and to analyze their antimicrobial susceptibility pattern.
\end{abstract}

M aterials and Methods: This is a retrospective analysisfrom January 2016 to December 2017. Urine samples collected in appropriate sterile manner were screened for polymorphonuclear leucocytes and bacteria by routine microscopic examination. This was followed by plating on MacConkey's agar and Blood agar. Isolated Acinetobacter strains which are oxidase negative and non-lactose fermenters from MacConkey's agar were identified with M atrix assisted laser desorption ionization time of flight mass spectrometry (MALDI-TOF MS) to confirm the identification. Antibiotic susceptibility was performed by VitekCompact ${ }^{\mathrm{TM}} 2$ (Biomeuriux, France) as per CLSI standards establishing M IC (M inimum Inhibitory Concentration).

Results : Of the 429 isolates of Acinetobacterspecies from urinary tract, Acinetobacterbaumannii complex (78\%) found to be the most common species followed by Acinetobacterjunii(10\%) and Acinetobacterbaumannii(8\%). Of the 334 (78\%) isolates of Acinetobacterbaumannii complex from urinary tract, $73(21.85 \%)$ isolates were resistant with doripenem, 62 (18.56\%) with imipenem and 59 (17.66\%) were resistant with meropenem with $\mathrm{MIC}$ values $>=8 \mu \mathrm{g} / \mathrm{ml}$. The rest of the isolates like Acinetobacterjunii, Acinetobacterjohnsonii, andAcinetobacterlwoffiiare found to be more sensitive with carbapenems with lower MIC values.

Discussion : The treatment of multidrug-resistant bacteria in hospitalized patients continues to be a challenge for the clinician's in routine practice. Acinetobacter baumannii complex has proven to be an important pathogen in health care associated infections with significant mortality and morbidity. The drug resistant nature of the pathogen and its unpredictable susceptibility patternsmake empirical and therapeutic decisions even more difficult.

Conclusion : A. baumanniiis an important opportunistic agent of nosocomial UTI, especially in patients with longer hospitalization, antibiotic exposure, urinary catheterization and decreased immunity. High antimicrobial resistance and patient co-morbidities limit therapeutic choices. Hence, alternative therapeutic options are urgently needed to treat a patient with $A$. baumanniiinfection.

\section{Introduction}

Acinetobacter species have emerged as important cause ofnosocomial infections like pneumonia and urinary tract infections. These species have been known to cause different kinds of opportunistic infections. Acinetobacter baumanii is now recognized to be the Acinetobacter 
genomic species of great clinical importance ${ }^{1}$. Acinetobacter species are areknown to be highly resistant to commonly used antimicrobial agents ${ }^{2}$. Acinetobacter species cause a wide variety of illness in debilitated and hospitalized patients especially in intensive care units (ICU). These bacteria survive for long time in the hospital environment. There is substantial increase inthe opportunity for cross infection ${ }^{3}$. Lower respiratory tract and urinary tract are more commonly affected sites with Acinetobacter species, and these distributions are very similar to that of other nosocomial pathogens ${ }^{4}$. As neonates are highly susceptible to infections with opportunistic pathogens, bactaeremia and septicemia due to Acinetobacter species is gaining importance in neonates ${ }^{5}$. Because of frequent resistance to aminoglycoside's, fluoroquinolone's, ureidopenicillin's and third generation cephalosporins, carbapenem are important agents in managing Acinetobacter infections. In the recent years there has been an alarming increase in carbapenem resistance in Acinetobacter species ${ }^{6,7}$. Since Acinetobacter species are ubiquitous and have tremendous colonizing capacity, it is difficult to explain the role of Acinetobacter acquisition in the ICU and medical wards. In addition, the risk factors for Acinetobacter acquisition may vary in different set up with epidemic, outbreak of infection or endemic colonization ${ }^{8}$. The risk factors that are involved in acquisition of Acinetobacter infection, as reported by other investigators, are assisted ventilation, use of broad spectrum antimicrobial therapy, endotracheal intubation, parenteral nutrition andurinary and intravascular catheterization. The objective of this study was to determine the frequency of urinary tract infection (UTI) caused by different Acinetobacter species in hospitalized patients and in the community and to analyze their antimicrobial susceptibility pattern.

\section{Materialsand Methods}

All the urine (midstream, catheterized and suprapubic aspiration) specimens collected from patients with suspected UTI were cultured on blood agar and MacConkey's agar in the Department of Microbiology,
Metropolis healthcare limited, Mumbai, India. During the study period (January 2016to December 2017), Gram negative, oxidase negative, non-lactose fermenting coccobacilliisolated in significant counts $\left(>1 \times 10^{5} \mathrm{cfu} / \mathrm{ml}\right.$ ) in pure culture were included in the study. UTI was defined as the presence of any one of the following symptoms: fever, burning, urgency, frequency of micturition, supra pubic tenderness and growth of $>=1 \times 10^{5} \mathrm{cfu} / \mathrm{ml}$ of Acinetobacter species from urine specimen. Patients with suprapubic aspiration, even less colony count $\left(<1 \times 10^{5} \mathrm{cfu} / \mathrm{ml}\right)$ with presence of polymorph nuclear leucocytes was considered significant and included in the study. The objective of this study was to determine the antibiotic susceptibility pattern of the isolated strains of Acinetobacter species with special reference to cephalosporin's and carbapenem resistance and to guide clinicians for appropriate antimicrobial therapy for reduction of morbidity \& mortality in hospitalized patients. This study was performed with patients admitted in a tertiary care hospital, developing symptoms of UTI at least after 48 hours of admission. Some patients from community acquired infection with symptoms of UTI have also been included in the study. Cases of urinary tract infection with established nonbacterial aetiology (fungal UTI) excluded from the study. Urine samples collected in appropriate sterile precautions were screened for pus cells and bacteria by routine microscopic examination. This was followed by plating on M acConkey's agar and Blood agar by $T$ streaking method with an inoculating loop of $4 \mathrm{~mm}$ diameter $(10 \mu$ l of un-centrifuged urine specimen). Inoculated plates were incubated overnight at $37^{\circ} \mathrm{C}$. Isolated Acineto bacter strains (Gram negative coccobacilli) which are oxidase negative and non-lactose fermenters from M acConkey's agar were identified with $M$ atrix assisted laser desorption ionization time of flight mass spectrometry (MALDI-TOF MS) to confirm the identification and speciation. Antibiotic susceptibility was performed by Vitek Compact $^{\mathrm{TM}} 2$ (Biomeuriux, France) as per CLSI guidelines establishing MIC (Minimum Inhibitory Concentration) of the tested antibiotics. 


\begin{tabular}{|c|c|c|c|c|}
\hline \multirow[t]{2}{*}{ Antimicrobial } & \multicolumn{3}{|c|}{$\mathrm{MIC} \mu \mathrm{g} / \mathrm{ml}$} & \multirow[t]{2}{*}{ Comments } \\
\hline & $\mathbf{S}$ & I & $\mathbf{R}$ & \\
\hline Doripenem & $<=2$ & 4 & $>=8$ & \multirow{2}{*}{$\begin{array}{l}\text { Breakpoints for doripenem are based on a dosage regimen of } \\
500 \text { mg every } 8 \mathrm{~h} \text {. }\end{array}$} \\
\hline & & & & \\
\hline Imipenem & $<=2$ & 4 & $>=8$ & \multirow{2}{*}{$\begin{array}{l}\text { Breakpoints for imipenem are based on a dosage regimen of } 500 \\
\text { mg every } 6 \mathrm{~h} \text {. }\end{array}$} \\
\hline & & & & \\
\hline M eropenem & $<=2$ & 4 & $>=8$ & \multirow{2}{*}{$\begin{array}{l}\text { Breakpoints for meropenem are based on a dosage regimen of } 1 \\
\text { g every } 8 \text { h or } 500 \text { mg every } 6 \mathrm{~h} \text {. }\end{array}$} \\
\hline & & & & \\
\hline Colistin & $<=2$ & - & $>=4$ & \multirow{5}{*}{$\begin{array}{l}\text { Colistinshould generally be given with a loading dose and at } \\
\text { maximum recommended doses, and used in combination with } \\
\text { other agents. Applies to A. baumanniicomplex only. (The only } \\
\text { approved MIC method for testing is broth micro dilution. Disk } \\
\text { diffusion and gradient diffusion should not be performed-2018 } \\
\text { CLSI). }\end{array}$} \\
\hline & & & & \\
\hline & & & & \\
\hline & & & & \\
\hline & & & & \\
\hline Polymyxin B & $<=2$ & - & $>-4$ & 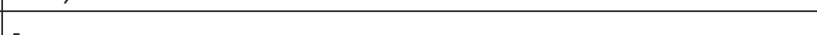 \\
\hline Gentamicin & $<=4$ & 8 & $>=16$ & \multirow[t]{2}{*}{-} \\
\hline Amikacin & $<=16$ & 32 & $>=64$ & \\
\hline Piperacillin-tazobactam & $<=16 / 4$ & $32 / 4-64 / 4$ & $>=128 / 4$ & \multirow[t]{5}{*}{-} \\
\hline Ceftazidime & $<=8$ & 16 & $>=32$ & \\
\hline Cefepime & $<=8$ & 16 & $>=32$ & \\
\hline Cefotaxime & $<=8$ & $16-32$ & $>=64$ & \\
\hline Ceftriaxone & $<=8$ & $16-32$ & $>=64$ & \\
\hline Ciprofloxacin & $<=1$ & 2 & $>=4$ & \multirow[t]{2}{*}{-} \\
\hline Levofloxacin & $<=2$ & 4 & $>=8$ & \\
\hline Trimethoprim-sulfamethoxazole & $<=2 / 38$ & - & $>=4 / 76$ & - \\
\hline
\end{tabular}

Table 1 : M inimum inhibitory concentrations of antimicrobials used for Acinetobacter species causing urinary tract infections adapted from CLSI. Performance Standards for Antimicrobial Susceptibility Testing 27th ed. CLSI supplement M 100. Wayne, PA: Clinical and Laboratory Standards Institute; 2017.

$\mathrm{S}=$ Susceptible; I=Intermediate; R=Resistant.

Results

\begin{tabular}{|c|c|c|c|c|c|c|c|c|c|c|c|c|c|c|c|}
\hline Organism $(\mathrm{N}=429) /$ & \multicolumn{3}{|c|}{ Acinetobacter } & \multicolumn{3}{|c|}{ Acinetobacter } & \multicolumn{3}{|c|}{ Acinetobacter } & \multicolumn{3}{|c|}{ Acinetobacter } & \multicolumn{3}{|c|}{ Acinetobacter } \\
\hline \multirow{3}{*}{ Antibiotic } & \multicolumn{3}{|c|}{ baumannii } & \multicolumn{3}{|c|}{ baumannii } & \multirow{2}{*}{\multicolumn{3}{|c|}{ junii }} & \multicolumn{3}{|c|}{ Iwoffii } & \multicolumn{3}{|c|}{ johnsonii } \\
\hline & Com & $\operatorname{ex~(N}$ & 334) & & $y=3$ & & & & & & $\mathrm{~N}=$ & & & $\mathrm{N}=$ & \\
\hline & $\mathbf{S}$ & $I$ & $\mathbf{R}$ & $\mathbf{S}$ & I & $\mathbf{R}$ & $\mathbf{S}$ & I & $\mathbf{R}$ & S & I & $\mathbf{R}$ & 5 & $I$ & $\mathbf{R}$ \\
\hline Doripenem & 260 & 1 & 73 & 30 & 0 & 6 & 39 & 1 & 2 & 7 & 0 & 1 & 6 & 1 & 2 \\
\hline Imipenem & 264 & 8 & 62 & 28 & 1 & 7 & 38 & 1 & 3 & 7 & 0 & 1 & 7 & 1 & 1 \\
\hline M eropenem & 269 & 6 & 59 & 29 & 1 & 6 & 40 & 1 & 1 & 7 & 0 & 1 & 7 & 1 & 1 \\
\hline Colistin & 327 & - & 7 & 34 & - & 2 & 42 & 0 & 0 & 8 & - & 0 & 9 & - & 0 \\
\hline Polymyxin B & 327 & - & 7 & 34 & - & 2 & 42 & 0 & 0 & 8 & - & 0 & 9 & - & 0 \\
\hline Gentamicin & 243 & 11 & 80 & 27 & 0 & 9 & 38 & 0 & 4 & 8 & 0 & 0 & 6 & 2 & 1 \\
\hline Amikacin & 245 & 7 & 82 & 23 & 2 & 11 & 36 & 1 & 5 & 8 & 0 & 0 & 6 & 1 & 2 \\
\hline $\begin{array}{l}\text { Piperacillin- } \\
\text { tazobactam }\end{array}$ & 246 & 6 & 82 & 24 & 0 & 12 & 36 & 2 & 4 & 8 & 0 & 0 & 7 & 1 & 1 \\
\hline Ceftazidime & 212 & 26 & 96 & 20 & 4 & 12 & 35 & 3 & 4 & 7 & 0 & 1 & $\frac{1}{7}$ & $\frac{1}{1}$ & $\frac{1}{1}$ \\
\hline Cefepime & 227 & 20 & 87 & 24 & 3 & 9 & 39 & 1 & 2 & 8 & 0 & 0 & 9 & 0 & 0 \\
\hline Cefotaxime & 223 & 18 & 93 & 21 & 2 & 13 & 36 & 1 & 5 & 7 & 0 & 1 & 7 & 1 & 1 \\
\hline Ceftriaxone & 228 & 8 & 98 & 21 & 1 & 14 & 36 & 1 & 5 & 7 & 0 & 1 & 7 & 1 & 1 \\
\hline Ciprofloxacin & 197 & 9 & 128 & 26 & 0 & 10 & 32 & 4 & 6 & 5 & 1 & 2 & 5 & 1 & 3 \\
\hline Levofloxacin & 205 & 8 & 121 & 28 & 0 & 8 & 34 & 3 & 5 & 6 & 1 & 1 & 5 & 1 & 3 \\
\hline $\begin{array}{l}\text { Trimethoprim- } \\
\text { sulfamethoxazole }\end{array}$ & 211 & - & 123 & 25 & - & 11 & 38 & - & 4 & 6 & - & 2 & 7 & - & 2 \\
\hline
\end{tabular}

Table 2 : Susceptibility pattern of different species of Acinetobacter from urinary tract isolates.

$\mathrm{S}=$ Susceptible; $\mathrm{I}=$ Intermediate; $\mathrm{R}=$ Resistant 


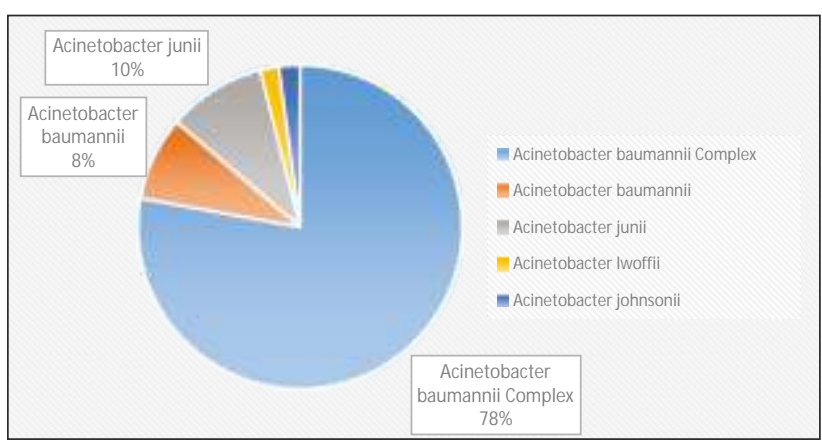

Graph 1 : Percentage proportion of different species of Acinetobacter isolated from urinary tract

Of the 429 isolates of Acinetobacter species from urinary tract, Acinetobacter baumannii complex (78\%) found to be the most common species followed by Acinetobacter junii(10\%) and Acinetobacter baumannii (8\%).

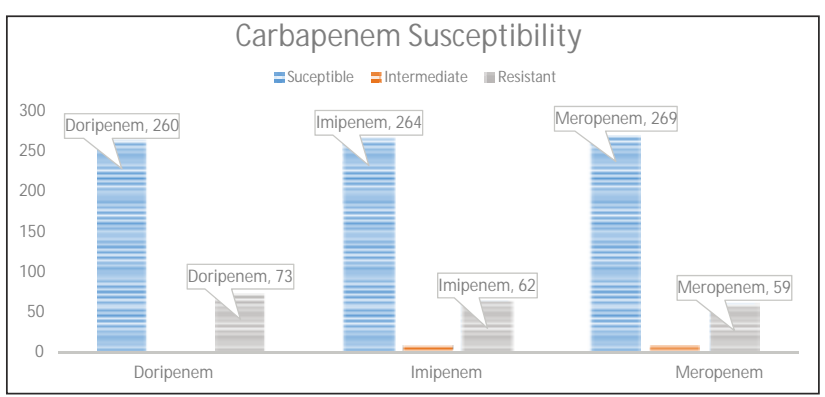

Graph 2 : Susceptibility of Acinetobacterbaumannii complex with carbapenems.

Of the 334 (78\%) isolates of Acinetobacterbaumannii complex from urinary tract, $73(21.85 \%)$ isolates were resistant with doripenem, 62 (18.56\%) with imipenem and 59 (17.66\%) were resistant with meropenem with MIC values $>=8 \mu \mathrm{g} / \mathrm{ml}$. The rest of the isolates like Acinetobacterjunii, Acinetobacterjohnsonii, andAcinetobacterlwoffiiare found to be more sensitive with carbapenems with lower MIC values.

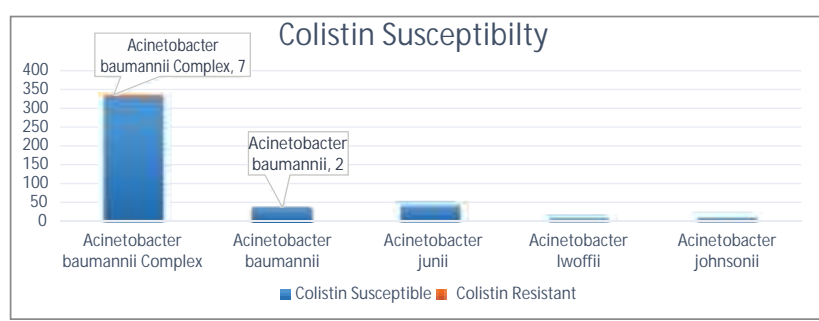

Graph 3 : Susceptibility of Acinetobacter specieswith polymixin B and colistin.

Of the 370 isolates of Acinetobacter baumannii and Acinetobacter baumannii complex, $9(2.43 \%)$ isolates were found resistant to both polymixin B and colistin with MIC $>=4 \mu \mathrm{g} / \mathrm{ml}$. Colistin should generally be given with a loading dose and at maximum recommended doses, and used in combination with other agents. Applies to A. baumanniicomplex only. The only approved MIC method for testing is broth micro dilution. Disk diffusion and gradient diffusion should not be performed.

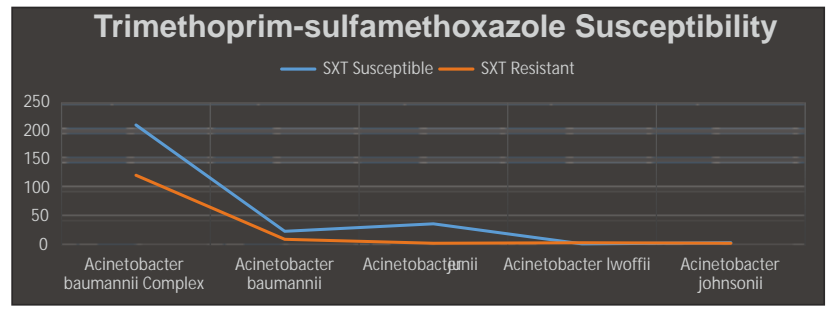

Graph 4 : Susceptibility of Acinetobacter specieswith Trimethoprim-sulfamethoxazole.

Of the 334 (78\%) isolates of Acinetobacter baumannii complex, $123(36.82 \%)$ isolates were resistant with Trimethoprimsulfamethoxazole. The rest of the isolates like Acinetobacterjunii, Acinetobacterjohnsonii, and Acinetobacter Iwoffii are found more sensitive with Trimethoprim - sulfamethoxazole.

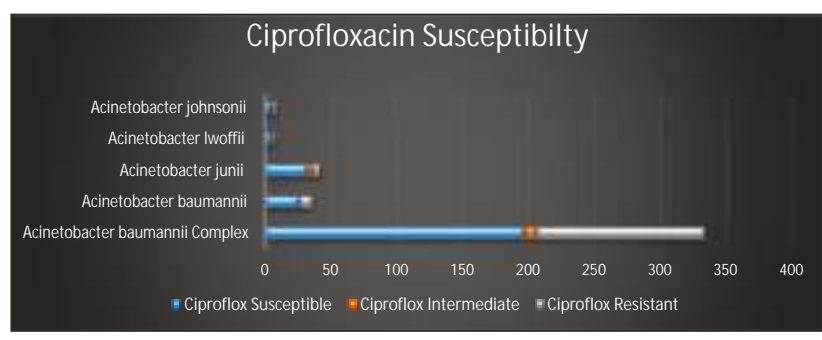

Graph 5: Susceptibility of Acinetobacter specieswith Fluoroquinolones.

Of the 334 (78\%) isolates of Acinetobacterbaumannii complex, $128(38.32 \%)$ isolates were resistant with ciprofloxacin and $121(36.22 \%)$ were resistant with levofloxacin. With the rest of the isolates like Acinetobacterjunii, Acinetobacterjohnsonii, andAcinetobacterlwoffii, there was no significant difference found between ciprofloxacin and levofloxacin susceptibility.

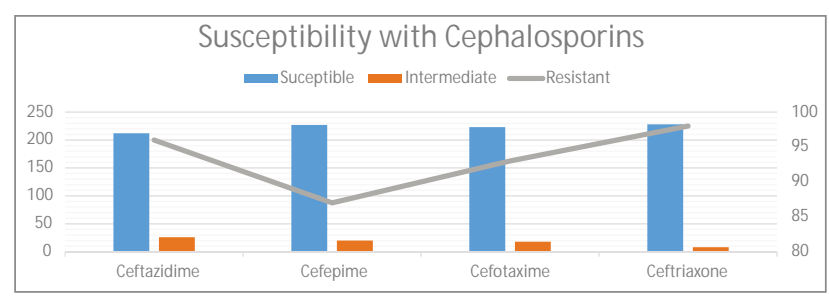

Graph 6: Susceptibility of Acinetobacterbaumannii complexwith cephalosporins.

Of the 334 (78\%) isolates of Acinetobacterbaumannii complex, 212 isolates were susceptible, 26 were intermediate and $96(28.74 \%)$ isolates were resistant with ceftazidime. Of the 334 (78\%) isolates of Acinetobacterbaumannii complex, 227 isolates were susceptible, 20 were intermediate and $87(26 \%)$ isolates were resistant with cefepime. In general, there was no significant difference in susceptibility was found between various cephalosporins like ceftazidime, cefotaxime, ceftriaxone and cefepime.

\section{Discussion}

The Acinetobacter calcoaceticus-Acinetobacter baumannii complex consists of four genotypically distinct but phenotypically very similar bacterial species or DNA groups: A. calcoaceticus (DNA group 1), A. baumannii (DNA 
group 2), unnamed ${ }^{9}$ DNA group 3and unnamed ${ }^{10}$ DNA group 13.The treatment of multidrug-resistant bacteria in hospitalized patients continues to be a challenge for the clinician's in routine practice. Acinetobacter baumannii complex has proven to be an important pathogen in health care associated infections with significant morbidity. The drug resistant nature of the pathogen and its unpredictable susceptibility patterns make empirical and therapeutic decisions even more difficult. The association of $A$. baumanniiwith pneumonia, bacteremia, and septicemia especially in neonates in ICU, wound infections, urinary tract infections, and meningitis has been well described as a nosocomial pathogen ${ }^{11}$. Risk factors associated with colonization or infection (which can be difficult to distinguish) include prolonged hospitalization, assisted ventilation, intensive care unit admission, recent surgical procedures, antimicrobial agent exposure, central venous catheter use, prior hospitalization, nursing home residence, and local colonization pressure on susceptible patients ${ }^{12,}{ }^{13}$.A. baumanniihas adapted to survive for extended periods on environmental surfaces. This phenomenon is of great important for transmission of organism within the health care setting. Health care associated infections with $\mathrm{A}$. baumannii are usually multidrug resistant due to prolonged exposure of antimicrobial agents and prolonged hospitalization. The impressive number of acquired mechanisms of resistance makes selection of an appropriate empirical antimicrobial agent exceedingly difficult. The mechanisms of drug of resistance in $\mathrm{A}$. baumanniinosocomial infections is beyond the scope of this study but can be reviewed in Peleg AY et al ${ }^{14}$.Degradation enzymes against $ß$-lactams splitting $ß$ lactam ring, modification enzymes against aminoglycosides like aminoglycosides modifying enzyme (AME), altered binding sites for quinolones, and a variety of efflux mechanisms and changes in outer membrane proteins have been reported. All of these mechanisms of drug resistance can be combined which may result in a highly drug-resistant pathogen, and at times pan-resistant opportunistic pathogen. Attributable mortality has been difficult to assess for an organism that appears to be as much a colonizer as it is a true pathogen. We do not know the pathogenicity of this organism causing urinary tract infections, but only those urine samples showing significant polymorphonuclear leucocytes (PMNL) and patients with suprapubic aspiration of urine have been included in the study. A. baumanniiis often identified in the sputum samples of patients with mechanical ventilation and in urinary catheterized patients. This quandary of pathogenicity has added to the difficulty of treating these highly resistant organisms, because many therapeutic strategies are associated with significant toxicity. The inhospital attributable mortality appears to be $8 \%-23 \%$, but for the intensive care unit, it was found to be $10 \%-43 \%{ }^{15}$. ${ }^{16}$.Strains of $A$. baumannii have acquired multiple drug resistance to newly developed antimicrobials in the last 30 years. These strains are known as multidrug resistant (MDR) A. baumannii. It is prevalent in many hospitals all over the world. Recently, it has been recognized as a leading nosocomial pathogen with significant mortality and morbidity in hospital settings ${ }^{17}$. Terminologies like multidrug resistant (MDR), extensive drug resistant (XDR), and pan-drug resistant (PDR) have been used with various definitions to describe the degree of antimicrobial resistance for Acinetobacter species. MDR Acinetobacter species can refer to being resistant to a minimum of three classes of antimicrobial agents e.g. all penicillins and cephalosporins fluoroquinolones, and aminoglycosides ${ }^{18}$. Another specific definition of multidrug resistance is whenever there is resistance to more than two of the following five drug classes: antipseudomonal cephalosporins (ceftazidime or cefepime), antipseudomonal carbapenems (imipenem or meropenem), ampicillin-sulbactam, fluoroquinolones (ciprofloxacin or levofloxacin), and aminoglycosides (gentamicin, tobramycin, or amikacin) ${ }^{19}$.MDR Acinetobacter strains which show additional resistant to carbapenems are defined as XDR. PDR Acinetobacter species is a term given to the XDR Acinetobacter species that is also resistant to polymyxins (polymyxin $B$ and colistin) and tigecycline. These categorizations help to define the extent of resistance and rational antimicrobial 
therapy in a clear way ${ }^{20}$.Enzymatic degradation by $\beta$ lactamases is the most prevailing mechanism of $\beta$ lactam resistance in $A$. baumannii. $\beta$ lactamases are divided into 4 molecular groups: Ambler class A, Ambler class B (metallo enzymes), Class $C \beta$ lactamases, and Ambler class $D$ (oxacillinases). These enzymes, at least partially, hydrolyze carbapenems along with other b-lactams ${ }^{21}$. Extendedspectrum B lactamases (ESBLs) from the Ambler class A group have been described for $A$. baumannii. Many ESBLS were identified in A. baumannii including TEM -92 and 116 from Italy and Netherlands respectively, and SHV-12 from China and the Netherlands. In addition, CTX-M -2 and CTXM-43 have been reported from Japan and Bolivia correspondingly. Seven different Penicillin binding proteins (PBPs) (1a, 1c, 2, 3, 4, 4b and 5) in A. baumannii were found. The resistance of $A$. baumanniito carbapenems is related to decreased drug uptake because of porin deficiency, and diminished affinity for the drug due to modification of the PBP's by mutations which is described by a reduced expression of PBP-2, as shown in isolates from Seville, Spain $^{22}$.Of the 334 (78\%) isolates of Acinetobacter baumannii complex, 212 isolates were susceptible, 26 were intermediate and $96(28.74 \%)$ isolates were resistant with ceftazidime. Of the $334(78 \%)$ isolates of Acinetobacter baumannii complex, 227 isolates were susceptible, 20 were intermediate and $87(26 \%)$ isolates were resistant with cefepime. In general, there was no significant difference in susceptibility was found between various cephalosporins like ceftazidime, cefotaxime, ceftriaxone and cefepime.Carbapenems (imipenem and meropenem) resistance Acinetobacter is increasingly reported, making M DR Acinetobacter infections difficult to treat. However, carbapenems continue to be the treatment of choice in cases where isolates are still susceptible to this antimicrobial class. Of the $334(78 \%)$ isolates of Acinetobacter baumannii complex from urinary tract, 73 $(21.85 \%)$ isolates were resistant with doripenem, 62 (18.56\%) with imipenem and 59 (17.66\%) were resistant with meropenem with MIC values $>=8 \mu \mathrm{g} / \mathrm{ml}$. The rest of the isolates like Acinetobacter junii, Acinetobacter johnsonii, and Acinetobacter Iwoffiiare found to be more sensitive with carbapenems with lower MIC values.Alteration in the structure of DNA gyrase or topoisomerase IV through mutations in the quinolones resistance-determining regions of the gyrA and parC genes is the main cause for resistance of $A$. baumannii to fluoroquinolones. These changes decrease the affinity of the quinolonesbinding to the enzyme-DNA complex. Another mechanismof resistance to the quinolones is caused by effluxsystems that reduce intracellular drug accumulation comprising RND-type pump Ade ABC and multi antimicrobial extrusion protein MATE pump AbeM . Until now, qur genes and plasmid mediated quinolonesresistance has not been reported for $A$. baumannii ${ }^{23}$. In the present study, of the 334 (78\%) isolates of Acinetobacter baumannii complex, 128(38.32\%) isolates were resistant with ciprofloxacin and $121(36.22 \%)$ were resistant with levofloxacin. With the rest of the isolates like Acinetobacter junii, Acinetobacter johnsonii, and Acinetobacter Iwoffii, there was no significant difference found between ciprofloxacin and levofloxacin susceptibility.Tigecycline derivative of minocycline and has bacteriostatic activity against MDR A. baumannii ${ }^{24}$. Highlevel resistance to tigecycline has been reported for some multidrug-resistant A. baumannii isolates, with a concern that this organism can quickly escape this antimicrobial mediated efflux pumps. Over expression of a multi-drug efflux pump in $A$. baumannii isolates with reduced susceptibility to tigecycline has been described ${ }^{25}$. Tigecycline is a good therapeutic option for treatment of life threatening MDR A. baumannii infections. Its role has been studied in bone and soft tissue infections, blood stream and gastrointestinal infections. However its antimicrobial activity in treating urinary tract infection is questionable. In the present study we did not test any isolate of $A$. baumannii complex against tigecycline. Amikacin and gentamicin are some of the aminoglycoside agents used as therapeutic options in cases of infection with multidrug-resistant $A$. baumannii isolates that retain susceptibility. These options are typically used in combination with another active antimicrobial agent. $M$ any multidrug-resistant $A$. baumannii isolates maintain 
an intermediate susceptibility to amikacin or gentamicin to which resistance is highly correlated with aminoglycoside modifying enzymes (AME) or efflux pump mechanisms ${ }^{26}$. Of the 334 isolates of A. baumannii complex 245(73.35\%) isolates were susceptible with amikacin and $82(24.55 \%)$ were resistant and $7(2 \%)$ isolates have shown intermediate susceptibility. There was no significant difference in susceptibility between amikacin and gentamicin. Other isolates of Acinetobacter like A. junii. A. johnsonii and A. Iw offiiwere found to be more sensitive to aminoglycosides but significance of these organisms in causing urinary tract infection needs to be further evaluated. Colistin, a cationic polypeptide. It is part of the polymyxin family (colistimethate or colistinsulfomethate or polymyxin E). It is an old potent broad spectrum antimicrobial agent. This agent was originally used in the $1960 \mathrm{~s}$ and 1970 . Because of concerns with nephrotoxicity and neurotoxicity, this agent was not prescribed frequently in clinical practice. Clinicians are going back to the use of polymyxin $B$ or polymyxin $E$ (colistin) for highly drug-resistant $A$. baumannii infections. Observational studies have shown a rate of $57-77 \%$ of cure or improvement among severely ill patients with multidrug-resistant $\mathrm{A}$. baumannii infections treated with colistin. These infections included pneumonia, bacteremia, sepsis, intra-abdominal, and Central Nerves System infection ${ }^{27}$. Of the 370 isolates of Acinetobacter baumannii and Acinetobacter baumannii complex, $9(2.43 \%)$ isolates were found resistant to both polymixin B and colistin with MIC $>=4 \mu \mathrm{g} / \mathrm{ml}$. Colistin should generally be given with a loading dose and at maximum recommended doses, and used in combination

\section{References}

1. Prashanth K, Badrinath S. Nosocomial infections due to Acinetobacter species: Clinical findings, risk factors and prognostic factors. Indian J Med M icrobiol 2006; 24:39-44.

2. Deep A, Singhi S. Nosocomial infections in PICU. Pediar Today 2004; 783-92.

3. Sinha M, Srinivasa H. Mechanisms of resistance to carbapenams in Meropenem resistant Acinetobacter isolates from clinical samples. Indian J Med M icrobiol 2007; 25:121-125.

4. Berogue-Berezin E, Towner KJ. Acinetobacter species as nosocomial pathogen:M icrobiological, clinical and epidemiological. ClinM icrobiol Rev 1996; 9:365-366.

5. Vinodkumar CS, Neelagund F. Acinetobacter septicemia in neonates. IndianJ Med Microbiol 2004; 22:71.

6. M anikal VM , Landman D, Saurma G, Oydna E, Lal H, Quale J. Endemic carbapenam-resistant Acinetobacter species in Brooklyn, New York: with other agents. As per recent CLSI guidelines 2018, colistin testing applies to $A$. baumanniicomplex only and no other Acinetobacter species. The only approved MIC method for colistin testing is broth micro dilution. Disk diffusion and gradient diffusion should not be performed. The nine strains of Acinetobacter which were found resistant with colistin were rechecked with Vitek Compatct $^{T M} 2$ automated susceptibility system (Biomerieux, France). However we did not do any manual broth micro dilution method for testing colistin resistance.

\section{Conclusion}

A. baumannii is an important opportunistic and emerging pathogen that can lead to serious nosocomial infections especially in patients with longer hospitalization, antibiotic exposure, urinary catheterization and decreased immunity. Its pathogenic potential includes the ability to adhere to surfaces, form biofilms, display antimicrobial resistance and acquire genetic material from unrelated genera, making it a versatile and difficult adversary to control and eliminate. High antimicrobial resistance and patient co-morbidities limit therapeutic choices. The optimal treatment for $\mathrm{A}$. baumannii, especially nosocomial infections resulting from multiple resistant strains, remains to be established. It is thus a clinical imperative that welldesigned procedures are put in place to help guide clinicians on decisions regarding the current best therapeutic practice.

\section{Acknowledgements}

We are thankful to the Department of Microbiology, M etropolis Healthcare Ltd, M umbai, India.

Citywide prevalence, inter-institutional spread and relation to antibiotic usage. Clin Infect Dis 2000; 31:101-106.

7. Bou G, Cervero G, Dominguez MA, Quereda C, Martinez-Beltran J. Characterization of a nosocomial outbreak caused by a multiresistant Acinetobacter baumanii strain with a carbapene-hydrolysing enzyme: High level carbapenem resistance in A.baumanii is not due solely to the presence of beta-lactamase. J ClinM icrobiol 2000; 38:3299-3305.

8. Forster DH, Daschner FD. Acinetobacter species as nosocomial pathogens. Eur J Clin M icrobiological Infect Dis 1998; 17:73-77.

9. Grimont F, Grimont PA. Ribosomal ribonucleic acid gene restriction patterns as potential taxonomic tools. Ann Inst Pasteur Microbiol. 1986 Sep-Oct; 137B (2):165-175.

10. Tjernberg I, Ursing J. Clinical strains of Acinetobacter classified by DNA-DNA hybridization. APM IS. 1989 Jul; 97(7):595-605

11. Maragakis LL, Perl TM. Acinetobacter baumannii: epidemiology, 
antimicrobial resistance, and treatment options. Clin Infect Dis 2008; 46(8):1254-1263.

12.Jang $T$, Lee $S$, Huang $C$, Lee $C$, Chen W. Risk factors and impact of nosocomial acinetobacterbaumanniiblood stream infections in the adult intensive care unit: a case-control study. J Hosp Infect 2009; 73: 143-150.

13. Mahgoub S, Ahmed J, Glatt AE. Underlying characteristics of patients harboring highly resistant Acinetobacter baumannii. Am J Infect Control 2002; 30(7):386-390.

14. Peleg AY, Seifert H, Paterson DL. Acinetobacter baumannii: emergence of a successful pathogen. ClinM icrobiol Rev 2008; 21(3):538-582.

15. Falagas ME, Rafailidis PI. Attributable mortality of Acinetobacter baumannii: no longer a controversial issue. Crit Care 2007; 11(3):134.

16. Falagas $M E$, Bliziotis IA, Siempos II. Attributable mortality of Acinetobacter baumanniiinfections in critically ill patients: a systematic reviewof matched cohort and case-control studies. Crit Care 2006; 10(2):R48.

17. Abbo, A., Navon-Venezia, S., Hammer-M untz, O., Krichali, T., SiegmanIgra, Y., Carmeli, Y., 2005. Multidrug-resistant Acinetobacter baumannii. Emerg. Infect. Dis. 11, 22-29.

18.Jung, J., Park, W., 2015. Acinetobacter species as model microorganisms in environmental microbiology: current state and perspectives. Appl. Microbiol. Biotechnol. 99, 2533-2548.

19. Peleg, A.Y., Seifert, H., Paterson, D.L., 2008. Acinetobacter baumannii: emergence of a successful pathogen. Clin. Microbiol. Rev. 21, 538-582.

20. Manchanda, V., Sanchaita, S., Singh, N., 2010. Multidrug resistant Acinetobacter. J. Global Infect. Dis. 2, 291.
21.Jain, R., Danziger, L.H., 2004. Multidrug-resistant Acinetobacter infections: an emerging challenge to clinicians. Ann. Pharmacother. 38, 1449-1459.

22. Perez, F., Hujer, A.M ., Hujer, K.M ., Decker, B.K., Rather, P.N., Bonomo, R.A., 2007. Global challenge of multidrug-resistant Acinetobacter baumannii. Antimicrob. Agents Chemother. 51, 3471-3484.

23. Potron, A., Poirel, L., Nordmann, P., 2015. Emerging broad-spectrum resistance in Pseudomonas aeruginosa and Acinetobacter baumannii: mechanisms and epidemiology. Int. J. Antimicrob. Agents 45, 568585.

24. Dinc, G., Demiraslan, H., Elmali, F., Ahmed, S.S., Alp, E., Doganay, M. 2015. Antimicrobial efficacy of doripenem and its combinations with sulbactam, amikacin, colistin, tigecycline in experimental sepsis of carbapenem-resistant Acinetobacter baumannii. N. Microbiol. 38, 67-73.

25. Eliopoulos, G.M., Maragakis, L.L., Perl, T.M., 2008. Acinetobacter baumannii: epidemiology, antimicrobial resistance, and treatment options. Clin. Infect. Dis. 46, 1254-1263.

26. Yadav, R., Landersdorfer, C.B., Nation, R.L., Boyce, J.D., Bulitta, J. B., 2015. Novel approach to optimize synergistic carbapenemaminoglycoside combinations against carbapenemresistant Acinetobacter baumannii. Antimicrob. Agents Chemother 59, 2286- 2298.

27. Vourli, S., Frantzeskaki, F., M eletiadis, J., Stournara, L., Armaganidis, A., Zerva, L., Dimopoulos, G., 2015. Synergistic interactions between colistin and meropenem against extensively drug-resistant and pandrug-resistant Acinetobacter baumannii isolated from ICU patients. Int. J. Antimicrob. Agents. 